\title{
Adverse effects of antiretroviral therapy or another disease?
}

\author{
Mariana Stanescu', Augustin Cupsa ${ }^{2}$, Cristiana Eugenia Simionescu ${ }^{3}$, \\ Adina Andreea Turcu ${ }^{1,4}$, Eugenia-Andreea Marcu' ${ }^{1}$, Florentina Dumitrescu ${ }^{1,2}$ \\ 1"Victor Babes" Hospital of Infectious Diseases and Pneumology, Craiova, Romania \\ ${ }^{2}$ Department of Infectious Disease, University of Medicine and Pharmacy, Craiova, Romania \\ ${ }^{3}$ Department of Pathology, University of Medicine and Pharmacy, Craiova, Romania \\ ${ }^{4}$ Department of Oral Health, Faculty of Dentistry, University of Medicine and Pharmacy, Craiova, Romania
}

\begin{abstract}
The immune reconstitution inflammatory syndrome (IRIS) is a paradoxical inflammatory response that can occur in patients infected with the human immunodeficiency virus (HIV) in the first weeks after initiating antiretroviral therapy (ART). It can be manifested either by exposing an underlying infection, or by aggravating the symptoms of an opportunistic infection already diagnosed and under treatment. We present the case of a 24-year-old patient, diagnosed in September 2019 with HIV infection category C3. Extrapulmonary tuberculosis with lymph node and probably splenic metastases. Bacteremia with Staphylococcus aureus. Normocytic hypochromic anaemia - medium form. Antibacillary therapy and ART were initiated (10 days after treatment for tuberculosis). After about 2 weeks from the initiation of ART, the patient complained of intense lumbar pain with irradiation in the lower limbs. IRIS (bone tuberculosis) or a bone adverse reaction to ART was suspected, but the imaging examination (MRI-lumbar spine) reveals a lumbosacral disc herniation, the patient continuing ART, antibacillary treatment, adding neurological treatment.

Conclusions. IRIS should be considered in patients diagnosed with HIV in late stages, in which the introduction of ART may be followed by the aggravation or occurrence of an opportunistic infection posing a problem of treatment and differential diagnosis.
\end{abstract}

Keywords: immune reconstitution inflammatory syndrome, HIV, tuberculosis, antiretroviral therapy

\section{INTRODUCTION}

The immune reconstitution inflammatory syndrome (IRIS) is a paradoxical inflammatory response that can occur in patients infected with human immunodeficiency virus (HIV) who receive antiretroviral therapy (ART) in the first weeks after starting therapy, due to the restoration of immunity to specific infectious or non-infectious antigens [1].

IRIS comes in two forms: IRIS "unmasking" refers to the appearance of an underlying infection, previously undiagnosed, shortly after the start of antiretroviral therapy (ART); IRIS "paradoxically" refers to the aggravation of a previously treated infection after the onset of ART. This syndrome has three rules: anything is possible, nothing is as it was in the era before ART, IRIS does not mean that ART has failed. It is often difficult to distinguish between symptoms related to the HIV infection and those caused by ART [1]. The overall incidence of IRIS remains unknown, being variable in relation to the stud- 
ied population and the incidence of opportunistic infections. Common favouring factors identified in the onset of IRIS include: male gender, young age, low CD4 (Lf) lymphocyte count at the initiation of ARV therapy as well as a low CD4/ CD8 ratio, rapid decrease in HIV-1 RNA after ART, and naïve status at the time of diagnosis of the opportunistic infection, the short interval between the onset of the treatment for opportunistic infections and the beginning of ART. Generally speaking, IRIS occurs in patients who have been initiated in ART during the first 2-3 months of TB therapy initiation, with a peak between 12-15 days, proving that the reaction is relatively quick, which requires precautions during this period of time [2]. Suppression of CD4 lymphocyte counts caused by HIV or immunosuppressive medication is associated with an immune response that is inefficient regarding certain infections. The benefits of ART therapy are proved by immunological monitoring (by rapid increase in CD4) and by virological reactions (decrease in HIV-1 viral plasma load). Despite such benefits, initiation of therapy is followed by secondary inflammatory response with clinical deterioration (general nonspecific symptoms such as fever) and impairment of certain organ function [3]. As exact estimates of incidence are not yet available and the spectrum of symptoms and etiology is variable, but increasing, in patients receiving ART, IRIS still remains a problem of diagnosis and treatment [4].

\section{MATERIALS AND METHODS}

We bring forth a 24-year-old patient from a rural area, without significant personal pathological history, who is admitted in an emergency at Drobeta-Turnu Severin County Hospital-Haematology Clinic for rectorrhagia, marked physical asthenia, weight loss (10 $\mathrm{kg}$ in 6 months) and dizziness.

\section{RESULTS}

Among the biological investigations Elisa-HIV 1,2 test is performed, with a positive result and the patient is transferred to the HIV/AIDS Department "Victor Babes" Hospital for Infectious Diseases and Pneumology Craiova.

The objective examination upon admission (September 2019) shows: affected general condition, afebrile, weight deficit $\left(\mathrm{BMI}=17.91 \mathrm{~kg} / \mathrm{m}^{2}\right.$ - body mass index), pale skin and mucous membranes, lingual fungal deposits, generalized micropolyadenopathy, tumour formation of approx. $1.5 \mathrm{~cm}$, well defined, clear outline, hard, mobile on the underlying planes, sensitive to palpation, located in the right lateral-cervical level, hypotrophic muscular system, poorly represented connective-adipose tissue, pulmonary stetacoustic: bilaterallyhardened vesicular murmur, $\mathrm{SaO}_{2}=93 \%$, ventricular rate $=83$ beats per minute, blood pressure $=110 / 74 \mathrm{mmHg}$, with abdominal movement during breathing, painful spontaneously and on palpation in the right hypochondrium and left hypochondrium, liver with margin less than $4 \mathrm{~cm}$ below the costal rim, grade II splenomegaly, external hemorrhoids, absent meningeal syndrome. Laboratory data show an inflammatory syndrome $(\mathrm{ESR}=120$ $\mathrm{mm} / 1 \mathrm{~h}, 140 \mathrm{~mm} / 2 \mathrm{~h}$ ), normocytic hypochromic anaemia-medium form $(\mathrm{HGB}=8.2 \mathrm{mg} / \mathrm{dl}, \mathrm{MCH}=27$ pg, $\left.\mathrm{MCV}=88 \mu \mathrm{m}^{3}\right), \mathrm{Ag} \mathrm{HBs}$-absent, HCV-absent Atc, Toxoplasma gondii Ig G antibodies, cytomegalovirus present, anti-HIV1,2-positive antibody Elisa test, HIV- Western Blot positive, HIV viral load = 755,000 copies/ml, CD4 = 131 cells $/ \mathrm{mm}^{3}$.

Pulmonary X-ray: nothing active at the pleuralpulmonary level.

Abdominal ultrasound: significant hepatomegaly right hepatic lobe $=17.3 \mathrm{~cm}$; splenomegaly -16.5 $\mathrm{cm} / 8 \mathrm{~cm}$, inhomogeneous spleen, with multiple hypoechogenous areas, diffusely contoured, infracentimetric; fluid areas in the right pleural cavity and pelvis.

Haematologic examination: lymphoproliferative syndrome (lymphoma under observation). Surgical consultation is recommended for lymph node biopsy with histopathological examination and chest-abdomen CT scan.

Stage diagnosis: HIV infection category $\mathrm{C} 3$ newly diagnosed case. Right lateral-cervical tumour formation (tuberculous lymphadenitis?lymphoma?). Normocytic hypochromic anaemia - medium form. External hemorrhoids.

Evolution and treatment: After lymph node biopsy (performed at the Surgery Clinic, Craiova County Emergency Clinical Hospital) the patient returns to the HIV AIDS Department with anaffected general condition, fever; right lateral-cervical scar (after lymph node biopsy), $\mathrm{SaO}_{2}=99 \%$, ventricular rate = 117 beats per minute, blood pressure $=102 / 56 \mathrm{mmHg}$. The biological picture shows leukocytosis with neu- 
trophilia, hypochromic normocytic anaemia-medium form, inflammatory syndrome (ESR $=140 \mathrm{~mm} / 1 \mathrm{~h}$, $160 \mathrm{~mm} /{ }^{2} \mathrm{~h}$ ), Staphylococcus aureus present in blood culture, hepatic cytolysis syndrome.

Chest computer examination with contrast agent reveals multiple lymphadenopathies, some with necrosis inside (supraclavicular, prevascular, superior and inferior paratracheal, subcarinal, hilar, axillary), fluid in moderate quantity in the pericardium, fluid in low quantity on the right pleural side, left pleural fluid layer without images of space-occupying processes in the lung areas.

The CT scan of the abdomen with contrast agent shows: spleen $12.84 / 9.70 \mathrm{~cm}$, inhomogeneous structure by the presence of multiple spontaneously
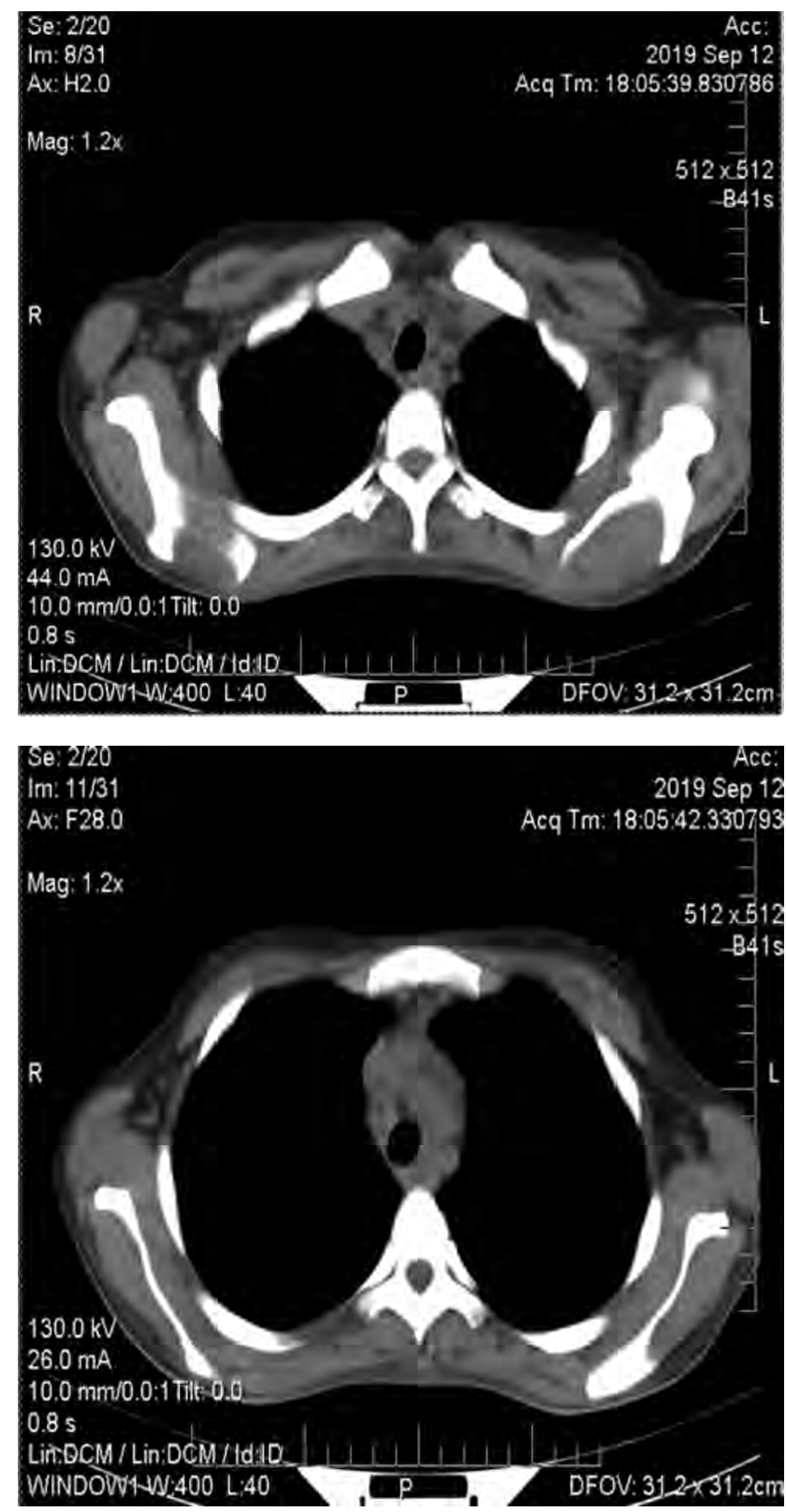

FIGURES 1A,B, C,D. Chest CT scan hypodense, neiodophilic formations, with a diameter between $9.2 \mathrm{~mm}$ and $17.3 / 19 \mathrm{~mm}$, disseminated on the entire splenic area, with the appearance of granulomatous metastases. There are no retroperitoneal lymphadenopathy or fluid in the peritoneal cavity.

As the patient's condition progressively worsens, she has fever episodes, antibacillarytreatment with Isoniazid + Rifampicin + Ethambutol + Ofloxacin is begun, 7/7 until the histopathological result is received. Approximately 14 days after the biopsy, the result of the histopathological examination is received, which highlights a lymph node with a distorted architecture by numerous epithelioid granulomas, with abundant necrosis and rare multinucleated cells of the Langhans type. Appearance of chronic granu-
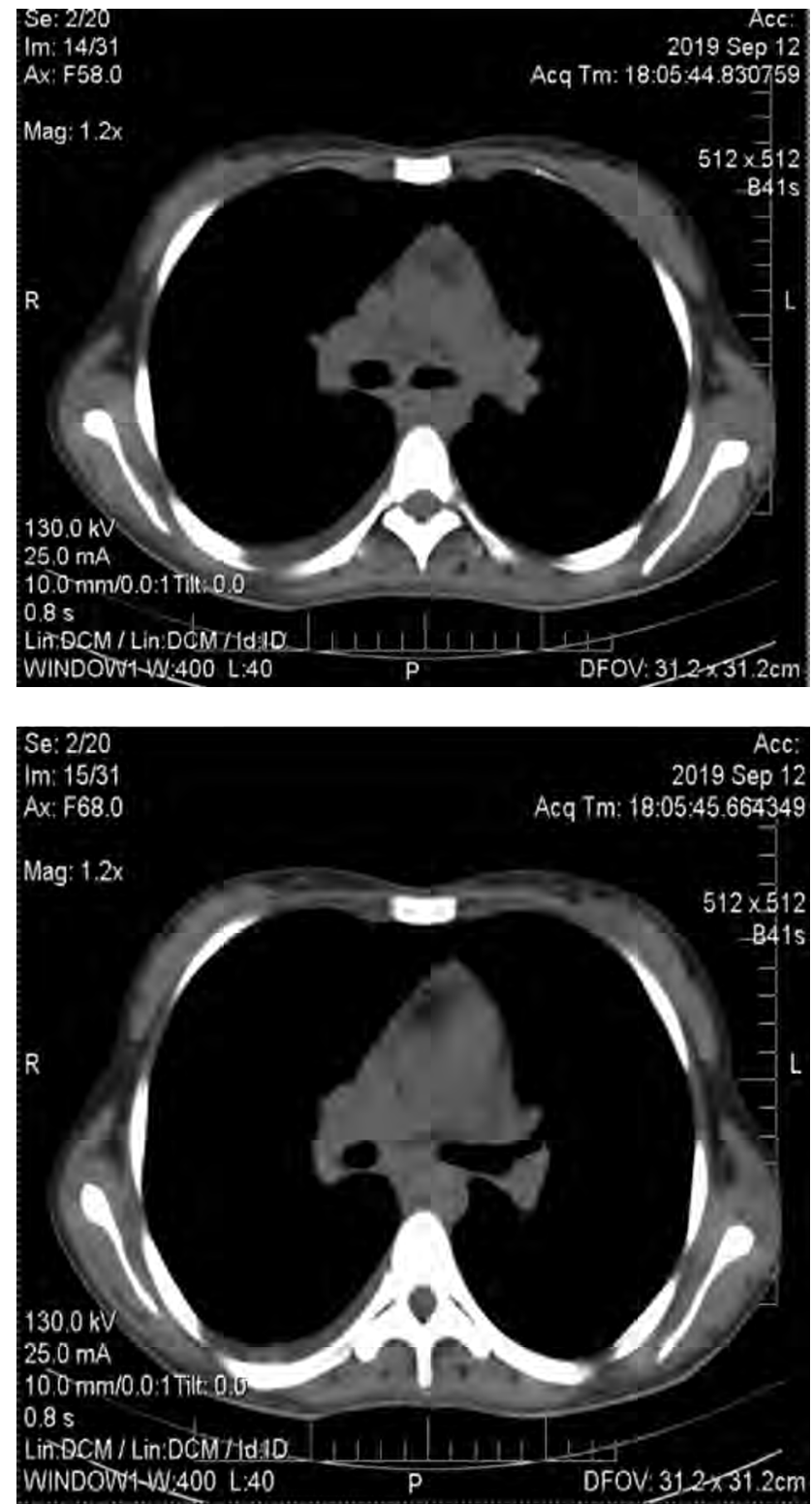


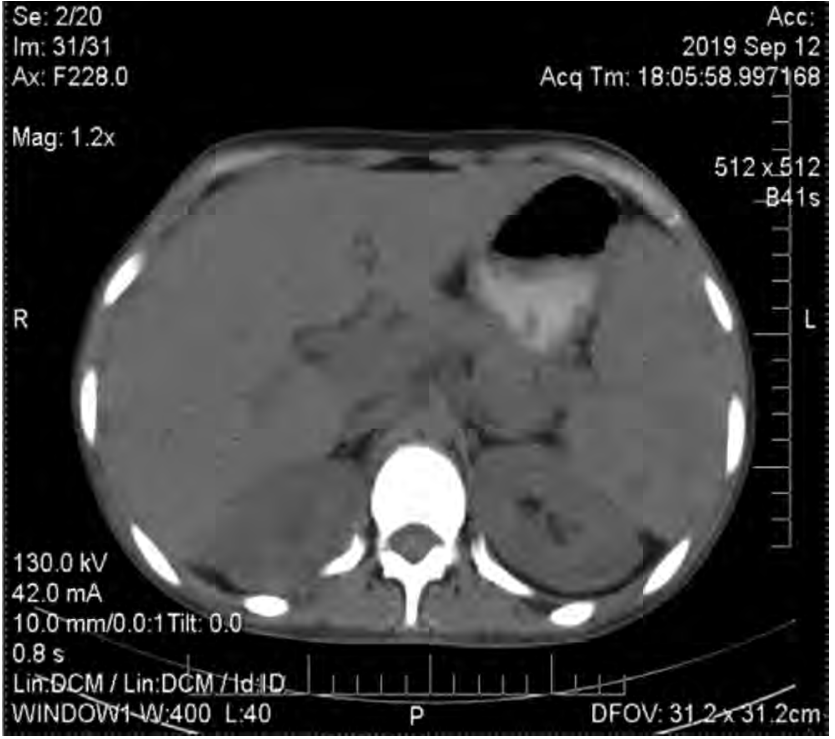

FIGURES 2A,B. Abdominal CT scan

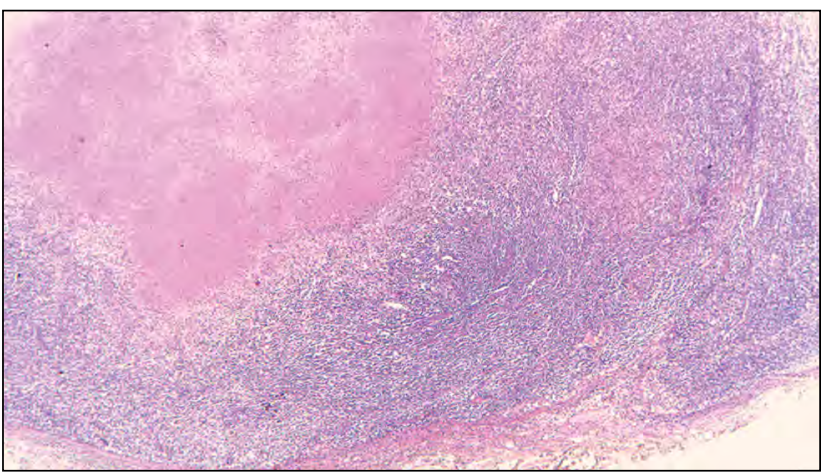

FIGURE 3A. Confluent epithelioid and caseous granulomas, HE col., x40

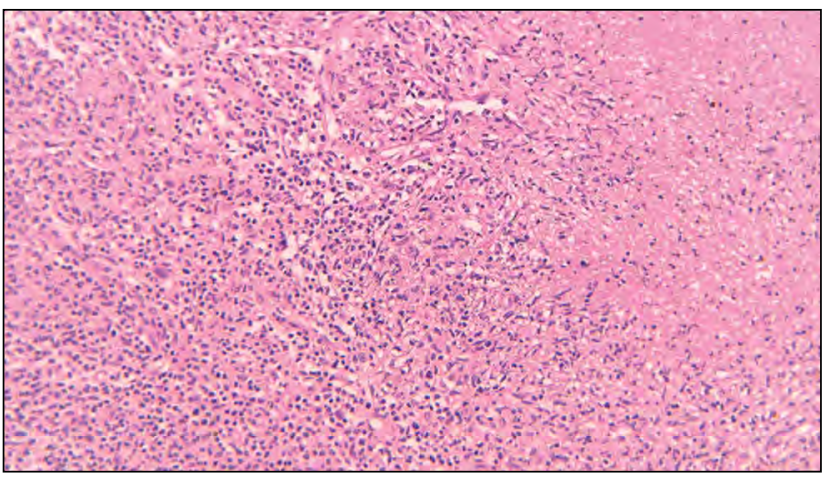

FIGURE 3C. Confluent epithelioid and caseous granulomas, $\mathrm{HE} \mathrm{col}$., $\times 100$, slice 2

lomatous adenitis, most likely of the tuberculosis (TB) type. Ziehl Nilson coloration highlights on the smear Mycobacterium tuberculosis.

Tenofovir + Emtriva + Tivicay $(\mathrm{TDF}+\mathrm{FTC}+$ DLG) antiretroviral therapyis initiated 10 days after initiation of the antibacillary therapy. During admission, after about 2 weeks from the initiation of ART, the patient complains of intense lumbar pain, with
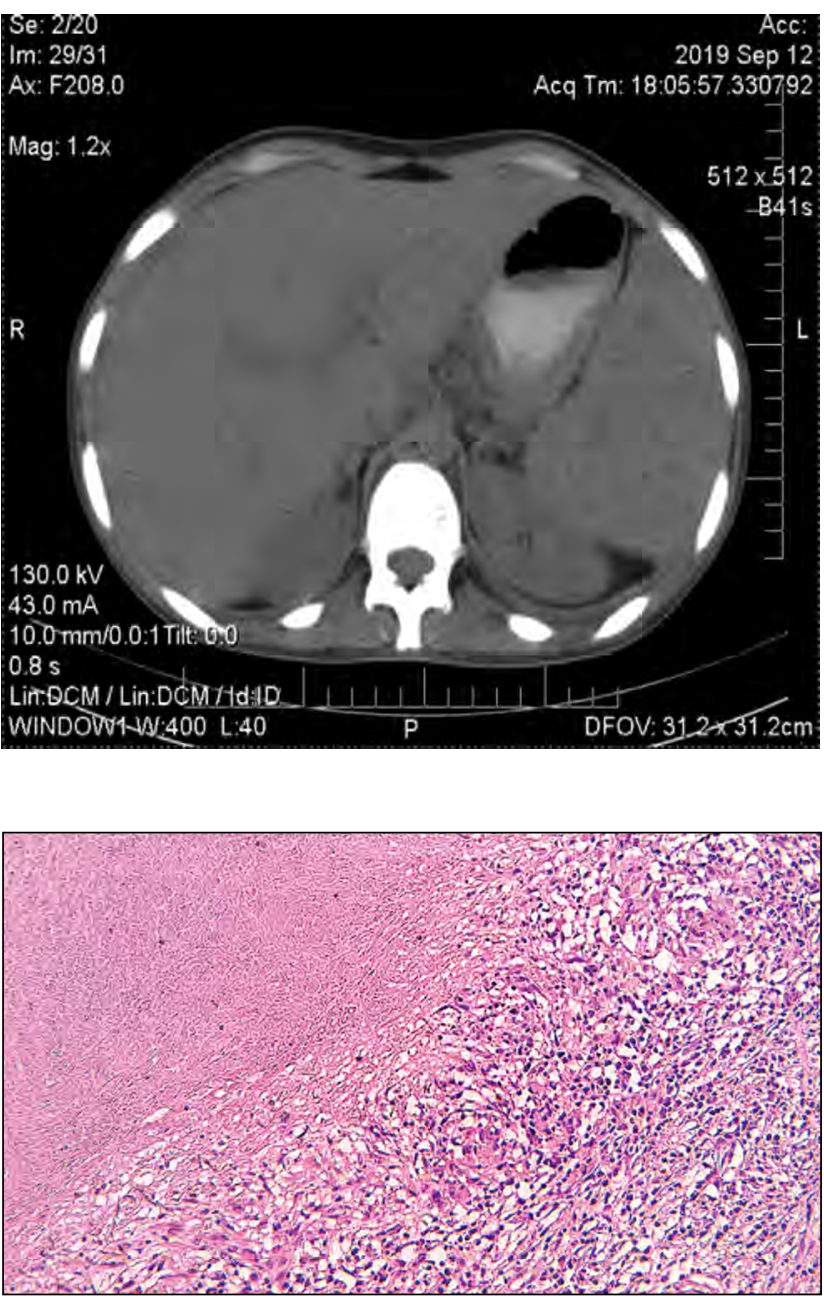

FIGURE 3B. Confluent epithelioid and caseous granulomas, HE col., x100, slice 1

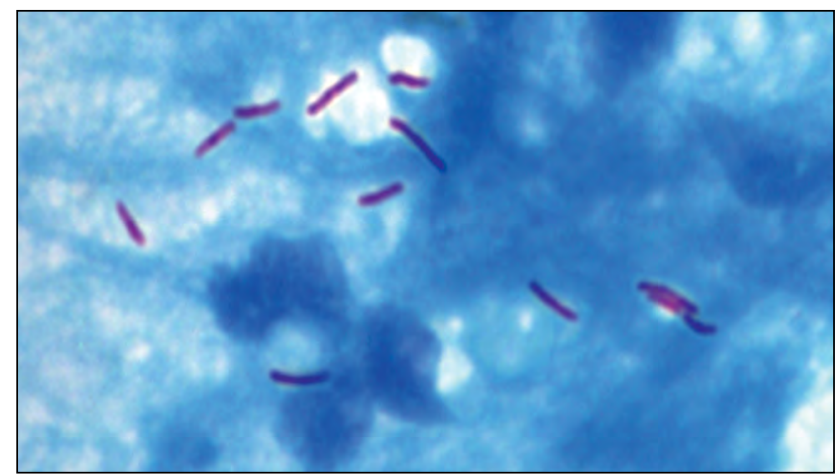

FIGURE 3D. Mycobacterium tuberculosis, Ziehl Nilson col., $x 1000$

irradiation in the lower limbs. Considering the patient's symptoms and associated pathology, IRIS, TB with bone metastasis is suspected.

CD4 lymphocyte count $=331$ cells $/ \mathrm{mm}^{3}$.

The patient underwent treatment with: antiretrovirals, antibacillary, corticotherapy (8 days), antibiotics (according to the antibiogram for Staphylococcus aureus) analgesics, gastric protectors and hepatoprotectants. 
However, the diagnosis of bone tuberculosis is refuted by the imaging examination (lumbar spine MRI - October 2019) which highlights a 14-L5, L5-S1 lumbosacral disc herniation.

The patient is discharged after 6 weeks, with an improved general condition, afebrile, balanced from the cardio-respiratory point of view, with partial remission of neurological symptoms, with the recommendation to continue ART, antibacillary treatment and to follow a physiotherapy program.

Diagnoses upon discharge: HIV infection category C3. Extrapulmonary tuberculosis with lymph node and probably splenic metastases. Bacteremia with Staphylococcus aureus. Lumb-sacral herniated disc L4-L5, L5-S1. Normocytic hypochromic anaemia medium form. Candida infections of the mouth. Seroreactive Toxoplasma gondii $\operatorname{IgG}+$. Seroreactive cytomegalovirus $\operatorname{IgG}+$.

\section{DISCUSSIONS}

Most studies regarding IRIS performed so far are retrospective and reported that $17-32 \%$ of patients who underwent ART developed IRIS, but the overall incidence of the syndrome remains unknown. A prospective study by David M. Murdoch et al., in South Africa on 423 ART-naïve HIV-infected patients, showed that 44 patients (10.4\%) developed IRIS in the first 6 months of therapy. The most common diagnoses included tuberculosis (41\%), followed by abscesses and folliculitis (18.2\%), varicella zoster $(13.6 \%)$, herpes simplex $(1 \%)$, cryptococcal meningitis $(6.8 \%)$, molluscum contagiosum $(6.8 \%)$ and Kaposi's sarcoma (4.5\%) [5]. However, discontinuation or delay of ART for 2 up to 6 months after TB therapy has been associated with high levels of mortality, making the decision not to initiate combination therapy quite difficult [6]. In a retrospective study (January 1, 2013 -December 31, 2017) conducted at the Regional Center for Monitoring and Evaluation of HIV/ AIDS Craiova, out of 110 patients with diagnosed TB, 5 cases presented TB-associated immune reconstitution inflammatory syndrome after the introduction of ART [7].

TB can occur in any number of CD4 T lymphocyte cells, although the risk increases with progressive immunosuppression. The emergence of antiretroviral therapy led to a decrease in the TB incidence among people with HIV infection, but nevertheless, even with the beneficial effects of ART, the risk of TB among people with HIV infection remains higher than that of the general population. Optimal management of HIV-related tuberculosis requires the resolution of both infections. HIV and TB co-treatment is complex due to the adherence requirements of multidrug therapy for two infections, drug interactions, overlapping profiles with side effects of anti-TB and ARV drugs and the IRIS risk. Simultaneous treatment of HIV and TB for co-infected patients improves survival (especially for people with CD4 count $<50$ cells $/ \mathrm{mm}^{3}$ ), decreases the risk of additional opportunistic diseases [8].

People infected with HIV have a lower bone density than uninfected people [9]. Higher loss of bone mineral density (DMO) was observed at the initiation of certain ART, in regimens containing TDF and some inhibitors of protease (IP). The clinical relevance of fracture risk has not been determined. Tenofovir alafenamide (TAF) associated fewer bone adverse events determined by the administration of tenofovir due to lower systemic exposure to this drug [10]. Switch studies from TDF to TAF suggest a possible recurrence of bone toxicity. However, long-term experience with TAF is lacking. Classic risk factors for osteopenia: old age, female gender, hypogonadism, family history of hip fracture, low BMI $\left(\leq 19 \mathrm{~kg} / \mathrm{m}^{2}\right)$, vitamin D deficiency, smoking, lack of physical activity, history of fractures to minimal trauma, excess alcohol ( $<3$ units/day), steroid exposure (minimum $5 \mathrm{mg} /$ day prednisone or equivalent to $>3$ months) [11].

In our patient's case, we eliminated an adverse reaction to TDF by using the imaging aspect (MRI-lumbar spine), with no osteopenia or osteolysis-type changes. Although IRIS could occur in this newly diagnosed patient with HIV-TB co-infection [12], it was a non-AIDS condition, a lumbosacral disc herniation.

\section{CONCLUSIONS}

IRIS should be considered in patients diagnosed with late-stage HIV, in whom the introduction of ART may be followed by the worsening or onset of anopportunistic infection. HIV-TB co-infection raises issues related to treatment and differential diagnosis in newly diagnosed patients with severe immunosuppression. 


\section{REFERENCES}

1. Hoffmann, Rockstroh. Managing Side Effects. HIV Book 2016. www. hivbook.com.

2. Murdoch DM, Venter WD, Van Rie A, Feldman C. Immune reconstitution inflammatory syndrome (IRIS): review of common infectious manifestations and treatment options. AIDS Res Ther. 2007;4:9.

3. French MA, Lenzo N, John M, Mallal SA, McKinnon EJ, James IR, Price P, Flexman JP, Tay-Kearney ML. Immune restoration disease after the treatment of immunodeficient HIV-infected patients with highly active antiretroviral therapy. HIV Med. 2000;1(2):107-115.

4. Mitrea M, Bîrluţiu V, Deac M. Sindromul de reconstrucție inflamatorie imună (IRIS). Acta Medica Transilvanica 2010;2(1):100-102.

5. Murdoch DM, Venter WD, Feldman C, Van Rie A. Incidence and risk factors for the immune reconstitution inflammatory syndrome in HIV patients in South Africa: A prospective study. AIDS. 2008; 22(5):601-610.

6. Lawn SD, Bekker LG, Miller RF. Immune reconstitution disease associated with mycobacterial infections in HIV-infected individuals receiving antiretrovirals. Lancet Infect Dis. 2005;5(6):361-373.

7. Florentina Dumitrescu, Augustin Cupşa, Andreea Cristina Stoian, Lucian Giubelan, Livia Dragonu, Mariana Stănescu, Irina Niculescu, Stefanita Diaconeasa. Tuberculosis in people living with HIVIAIDS.
Zilele Matei Balș. 2018, a XIV-a ediție a Zilelor Științifice ale Institutului Național de Boli Infecțioase „Prof. Dr. Matei Balș“, 31 oct-2 noiembrie 2018, volum de rezumate.

8. Guidelines for the Prevention and Treatment of Opportunistic Infections in Adults and Adolescents with HIV. Mycobacterium tuberculosis Infection and Disease. September 27, 2019. Available at http://www. aidsinfo.nih.gov/guidelines/html/4/adult-and-adolescentoi-prevention-and-treatment-guidelines.

9. Loiseau-Peres S, Delaunay C, Poupon S et al. Osteopenia in patients infected by the HIV. A case control study. Joint Bone Spine 2002;69:482-5.

10. Bone damage: screening and diagnosis. Available at https://www. eacsociety.org/files/guidelines-8.1.

11. Chowta MN, Kamath, Ramapuram JT, Shenoy KA, Hadigal S. Evaluation of Adverse Drug Reaction Profile of Drugs Used as First-Line Antiretroviral Therapy. Interdisciplinary Perspectives on Infectious Diseases 2018;2018:8095609.

12. Price $P$, Mathiot $N$, Krueger $R$, Stone $S$, Keane NM, French MA. Immune dysfunction and immune restoration disease in HIV patients given highly active antiretroviral therapy. Journal of Clinical Virology 2001;22(3):279-287. 